\title{
Sjögren's Syndrome - Oral Changes, Advanced Diagnosis, and Management - A Case Report
}

\author{
Minic $\mathrm{I}^{{ }^{1}}$, Pejcic $\mathrm{A}^{1}$, Pesic $\mathrm{Z}^{2}$, Zivkovic $\mathrm{V}^{3}$, Ilic $\mathrm{I}^{3}$ and Zarev $\mathrm{M}^{2}$ \\ ${ }^{1}$ Department of Periodontology and Oral medicine, Medical Faculty, University of Niš, Serbia \\ ${ }^{2}$ Department of Maxillofacial Surgery, Faculty of Medicine, University of Niš, Serbia \\ ${ }^{3}$ Department for Pathology, Faculty of Medicine, University of Niš, Serbia
}

*Corresponding author: Minic I, Department of Periodontology and Oral medicine, Medical Faculty, University of Niš, Serbia, E-mail: ivanminic@yahoo.com

Citation: Minic I, Pejcic A, Pesic Z, Zivkovic V, Ilic I, et al. (2016) Sjögren's Syndrome - Oral Changes, Advanced Diagnosis, and Management - A Case Report. J Case Rep Stud 4(4): 405. doi: 10.15744/23489820.4.4405

Received Date: April 21, 2016 Accepted Date: August 29, 2016 Published Date: August 31, 2016

\begin{abstract}
Sjögren syndrome (SS) is chronic, systemic autoimmune disease characterized by lymphocytic infiltration of the exocrine glands. It is an elaborate involvement of the lacrimal and salivary glands, which eventually lead to keratoconjunctivitis sicca and xerostomia. It may occur in two forms - Primary- occurs by itself and secondary, which is associated with another autoimmune disease, most commonly rheumatoid arthritis. Oral implications of SS are hyposalivation, xerostomia, inflamed and burning oral mucosa, rampant caries, sclerosis or growth of parotid gland, frequent manifestation of erythematous candidosis, angular cheilitis, increased plaque retention, and difficulty in swallowing. SS diagnosis is not easy. In addition to the many tests most important diagnostic method is a biopsy of the salivary gland. Early diagnosis is important to prevent further complications. The aim of this paper is to emphasis on oral changes, advanced diagnosis, and management of SS.
\end{abstract}

Keywords: Sjogren’s syndrome; Autoimmune Diseases; Salivary Gland Biopsy

\section{Introduction}

Sjögren's syndrome (SS) is a chronic systemic disorder characterized by polyglandular tissue destruction that causes keratoconjunctivitis sicca (KCS) and xerostomia (dry mouth). Patients with primary SS have KCS and xerostomia, whereas those with secondary SS have KCS, xerostomia and an autoimmune disease. Sjögren's syndrome can affect any age but the onset is most common in middle age or older, and $2-5 \%$ of people aged 60 years and above have primary SS [1,2].

The disease affects predominantly middle-aged women in the peri- or post-menopausal period. In addition to the primary syndrome, $30 \%$ of patients with rheumatoid arthritis, systemic lupus erythematosus and systemic sclerosis suffer secondary SS $[3,4]$.

The aetiology and pathophysiology of SS are still unknown. Autoimmunologic factors such as multiple autoantibodies particularly SS-A and SS-B, and a genetic predisposition are associated with SS. Some human leukocyte antigens also increase the risk of SS. Viral infection could be involved in the induction of SS. Epstein-Barr virus, human T lymphotrophic virus-1, human herpes virus, human immunodeficiency virus-1, hepatitis C virus, and cytomegalovirus may play a role [5-7]. The prognosis of SS is generally better than that of other autoimmune diseases.

Oral implications of SS are hyposalivation, xerostomia, inflamed and burning oral mucosa, rampant caries, sclerosis or growth of parotid gland, frequent manifestation of erythematous candidosis, angular cheilitis, increased plaque retention, and difficulty in swallowing $[8,9]$. Due to xerostomia and burning of the oral mucosa, patients with SS experience great discomfort and pain when wearing traditional removable prostheses.

Early diagnosis is important to prevent further complications. The aim of this paper is to emphasis on oral changes, advanced diagnosis, and management of Sjögren's syndrome.

\section{Case Report}

A 50 year old female patient reported to the Department of Oral Medicine, Medical Faculty, University of Nish, complaint of dryness of oral cavity and eyes since 2 years. Further history revealed difficulty in eating, adhering of food to the oral mucosa, urge of frequent sipping of water during eating and bilateral facial swelling since two years. 
On an inspection-swelling on right and left of the parotid gland, measuring about $2 \mathrm{~cm} \times 1,5 \mathrm{~cm}$ in diameter. Surface texture over the swelling was normal. Swelling extending superior up to the inferior border of the mandible at the angle region. Ear lobe was everted on the right side. It was diffuse, firm, non-movable, warm, tender on palpation, overlying surface texture was normal. Along with that dryness of eyes, fever was also noted. Bilateral submandibular lymph nodes were palpable.

Intraoral examination revealed extensive cervical caries, root stumps, atrophic tongue, buccal mucosa was thin and friable.

On the basis of medical history and clinical examination we suspected that it was a Sjögren syndrome but it was necessary to do additional diagnostic methods to confirm our suspicions. RA factor was positive. Minor salivary gland biopsy remains a highly used diagnostic procedure for the salivary component of SS. This is usually performed on the internal face of the lower lip on normal-appearing mucosa.

Under local anaesthesia an incision of around 1.5 to $2 \mathrm{~cm}$ is made between midline and the commissure, through the mucosa with penetration of the epithelium. With this procedure, usually 5 or more minor salivary glands are excised. The biopsy contributes towards the diagnosis of SS if the histopathological examination reveals a mononuclear infiltration with periductal or perivascular distribution.

The inflammatory infiltrate is quantified and a cluster of $\geq 50$ lymphocytes is termed a focus. The numbers of focus in an area of $4 \mathrm{~mm}^{2}$ of tissue surface render the focus score. A focus score $\geq 1$, as according to both the Revised International Classification Criteria, are considered positive for SS diagnosis. The histopathologic examination revealed numerous salivary gland acini and ducts in deeper stroma invaded by dense chronic inflammatory cells predominantly lymphoblasts with destruction of salivary gland architecture with focal periductal lymphocytic infiltrate (focus score: $\geq 1, \geq 50$ lymphocytic focus per $4 \mathrm{~mm}^{2}$ of glandular tissue) compared with negative histopathologic findings of the salivary glands for SS (Figure 1 and 2). Schirmer test and Rose Bengal dye test was positive. Serum immunoglobulin-SSA/RO and SSB/La positive for Sjögrens syndrome. ANA (antinuclear antibodies) was positive.

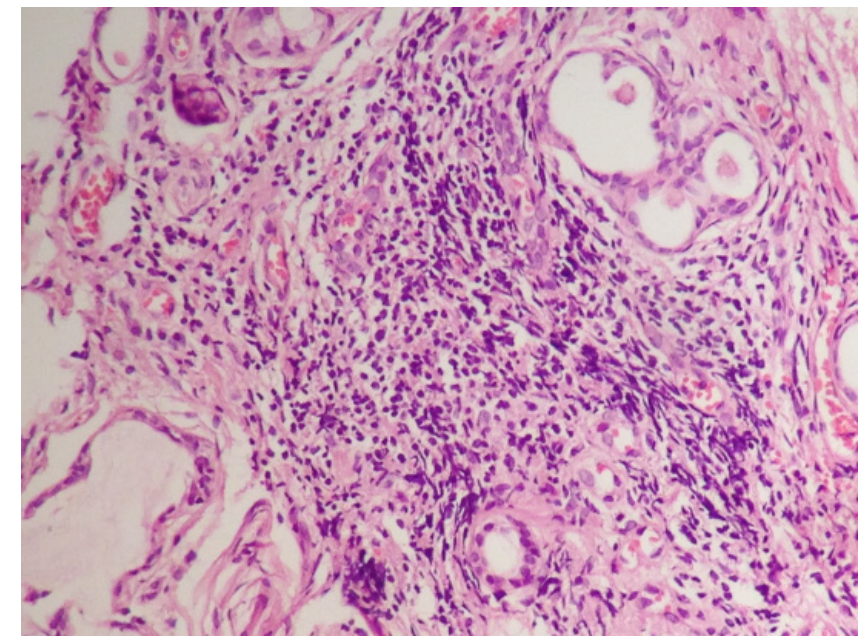

Histopathologic view of the minor labial salivary gland, typical for Sjögren's syndrome. Dense, well defined foci of lymphocytes replacing acini indicating acinar atrophy. A small island of residual salivary parenchyma remains around the periphery of the lobule Figure 1: Positive biopsy of salivary glands for Sjogren syndrome

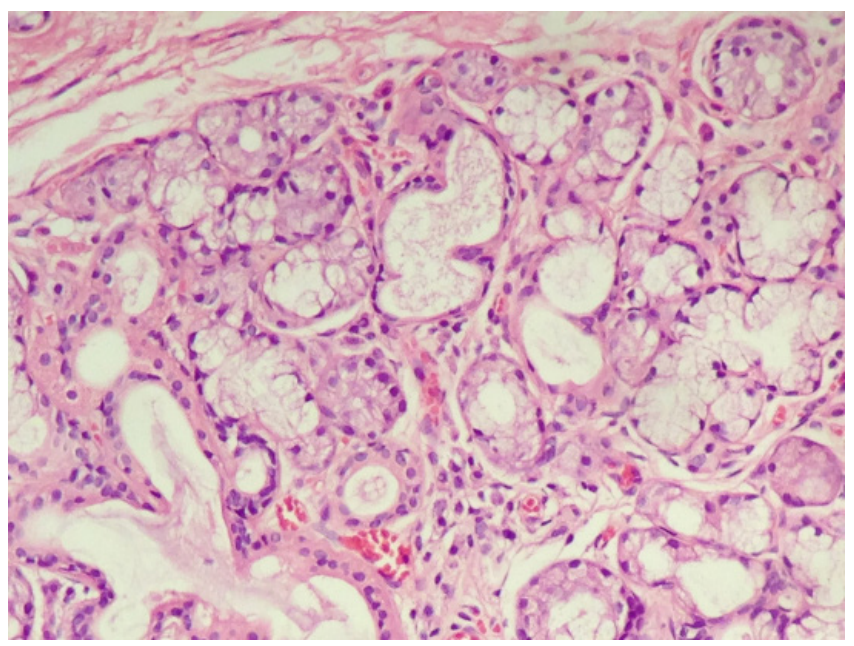

Figure 2: Healthy tissue of salivary gland with no signs of Sjögren syndrome 
Based on medical history, clinical examination and histopathological findings was diagnosed SS.

The patient was advised Tablet Pilocarpine $5 \mathrm{mg}, 4$ times/day chewable Vitamin C lozenges, artificial saliva Bioxtra for oral dryness and Pilocarpine 2\% eye drops for ocular dryness. The restoration of the carious teeth was done along with diet modification (in terms of intake of semisolid food and excessive liquids). Patient reported after a week with approximately 50\% reduction in ocular dryness and $50 \%$ reduction in oral dryness and burning sensation. Since then the patient is under regular follow up for observation.

\section{Discussion}

Sjogren's syndrome, a well known autoimmune disease, often affects women in fourth or fifth decades of life [10]. Prevalence of primary Sjogren's syndrome in the general population has been estimated to be around 1 to $3 \%$ [11]. In order to prove an updated literature review, we evaluated all past and recent series and case reports of primary Sjogren's syndrome and identified 3.9/100,000 cases overall in the world. It is reported that the enlargement of the major salivary glands occurs in 25-66\% of primary Sjogren's syndrome patients but is uncommon in patients with secondary Sjogren's syndrome [12]. Our patient also exhibited persistent swelling of the parotid glands, dryness of mouth and eyes since two years. Sjogren's syndrome has a wide clinical and immunologic spectrum and may progress from an exocrinopathy to a systemic disease with a variety of extraglandular manifestations and/ or associated autoimmune diseases. The difficulty in diagnosis is reflected on continuous review attempts of the 7 classification criteria that have been created in the past 25 years [13]. However, histological analysis of labial salivary gland biopsy (LSGB) is mostly a method of great importance according to the American-European Group Consensus (AEGC) criteria [14]. The characteristic findings of minor salivary gland biopsy in a person with Sjögren syndrome include the following $[15,16]$. The biopsy shows focal aggregates of at least 50 lymphocytes (predominantly CD4 $4^{+}$cells), and, to a lesser extent, plasma cells and macrophages, more than 1 focal aggregate is seen per $4 \mathrm{~mm}^{2}$, normal acini are replaced by lymphocytes, focal aggregates are seen in almost all glands, large foci are present, possibly showing germinal centers.

Epimyoepithelial islands are uncommon in the minor salivary gland but can be seen in the major salivary glands (in this case also Epimyoepithelial islands were not seen). Direct measurement of salivary flow (sialometry), radiocontrast assessment of salivary ductal system (sialography), and functional evaluation of the rate and density of salivary gland uptake of 99Tcmpertechnetate (scintigraphic isotope scanning) are primarily used in clinical trials, rarely to confirm the diagnosis in routine clinical practice $[17,18]$. Schirmer's Test is a strong indicator of diminished tearing [16].

In our paper we present a clinical case of SS, based on biopsy analysis we found lymphocytic infiltration and destruction of architecture themselves gland. Histopathologically, minor labial salivary gland biopsy revealed a lymphocytic score of $\geq 1$ in a periductal location, which according to criteria of Daniels and Whitcher, supports diagnosis of Sjögren's syndrome [5].

The treatment of patients with SS is directed toward the particular areas of the body that are involved and complications, such as infection. Dry mouth can lead to an increased rate of bacterial infections in the mouth and the need for medications [5,19]. Although artificial saliva and pilocarpine tablets are recommended, optimum oral hygiene and patient motivation are essential to prevent dental decay and oral mucosal infections. Dryness of the mouth can be relieved to some degree by providing artificial saliva. Cholinesterase inhibitors such as pilocarpine are sometimes recommended to stimulate salivary secretion but any benefits may be conterbalanced by side-effects such as nausea, diarrhea and bradycardia. Dryness of the eyes is treated with artificial tears such as methyl cellulose solution. In dentate patients, sweet-eating should be prohibited, and a high standard of oral hygiene should be maintained [20,21].

\section{Conclusion}

Dentists may often be the first to identify patients suffering from Sjögren's syndrome. Histological analysis of labial salivary gland biopsy is mostly a method of great importance. Treatment of Sjögren's syndrome depends on the extent and severity of the clinical manifestations and is better instituted through a multidisciplinary approach.

\section{References}

1. Daniels TE (1996) Sjögren’s syndrome: clinical spectrum and current diagnostic controversies. Adv Dent Res 10: 3-8.

2. Scully C, Felix HD (2005) Update for the dental practitioner, dry mouth and disorders of salivation. Br Dent J 199: 423-7.

3. Lynch MA (1979) Burket's oral medicine, diagnosis and treatment. Am J Ortho Dento Ortho 75: 106.

4. Mathews SA, Kurien BT, Scofield RH (2008) Oral manifestations of Sjögren’s syndrome. J Dent Res 87: 308-18.

5. Nikitakis NG, Rivera H, Lariccia C, Papadimitriou JC, Sauk JJ (2003) Primary Sjögren syndrome in childhood: report of a case and review of the literature. Oral Surg Oral Med Oral Pathol Oral Radiol Endod 96: 42-7.

6. Kassan SS, Moutsopoulos HM (2004) Clinical manifestations and early diagnosis of Sjögren syndrome. Arch Intern Med 164: 1275-84.

7. Manthorpe R (2002) Sjogren's syndrome criteria. Ann Rheum Dis 61: 482-4.

8. Carsons S (2001) A review and update of Sjogren's syndrome: manifestations, diagnosis, and treatment. Am J Manag Care 7: S433-43.

9. Daniels TE (1989) Clinical assessment and diagnosis of immunologically mediated salivary gland disease in Sjogren's syndrome. J Autoimmun 2: 529-41.

10. Lash AA (2001) Sjogren’s Syndrome: Pathogenesis, Diagnosis, and Treatment. Nurse Pract 26: 53-8. 
11. Manoussakis MN (2001) Sjogren's Syndrome. Orphanet encyclopedia 1-8.

12. Porkodi R, Rukmangatharajan S, Kanakarani P, Parthiban M, Vasanthy N, et al. (2003) Primary Sjogren's syndrome - Clinical and immunological features. J Indian Rheumatol Assoc 11: 63-5.

13. Baldini C, Talarico R, Tzioufas AG, Bombardieri S (2012) Classification criteria for Sjögren's syndrome: a critical review. J Autoimmun 39: 9-14.

14. Vitali C, Bombardieri S, Jonsson R, Moutsopoulos HM, Alexander EL, et al. (2002) Classification criteria for Sjögren's syndrome: a revised version of the European criteria proposed by the American-European Consensus Group. Ann Rheum Dis 61: 554-8.

15. Pesaramelli K, Anishetty R, Sravani K (2010) Sjogren's syndrome: a review of clinical features, diagnosis and treatments available. Int J Pharma Sci Rev Res 5: 93-9.

16. Božinović MT, Katić V, Krasić D, Veselinović D, Jovanović P, et al. (2009) Clinical, histopathological and immunohistological study of lymphoid disorders in the parotid gland of patients with Sjögren's syndrome. Vojnosanit Pregl 66: 955-60.

17. Delaleu N, Jonsson R, Koller MM (2005) Sjögren's syndrome. Eur J Oral Sci 113: 101-13.

18. García-Carrasco M, Fuentes-Alexandro S, Escárcega RO, Salgado G, Riebeling C, et al. (2006) Pathophysiology of Sjögren’s syndrome. Arch Med Res 37: 92132.

19. Harris E, Budd R, Genovese M, Firestein G, Sargent J, et al. (2005) Sjögren's Syndrome. In: Kelley's textbook of rheumatology (7 $7^{\text {th }}$ Edn) Vol. 2, Elsevier Saunders, USA.

20. Papas AS, Sherrer YS, Charney M, Golden HE, Medsger TA, et al. (2004) Successful treatment of dry mouth and dry eye Symptoms in Sjogren's syndrome patients with oral pilocarpine: A randomized, placebo-controlled, dose-adjustment study. J Clin Rheumatol 10: 169-77.

21. Hayashi Y, Arakaki R, Ishimaru N (2003) The role of caspase on the development of primary Sjögre’s syndrome. J Med Invest 50: $32-8$.

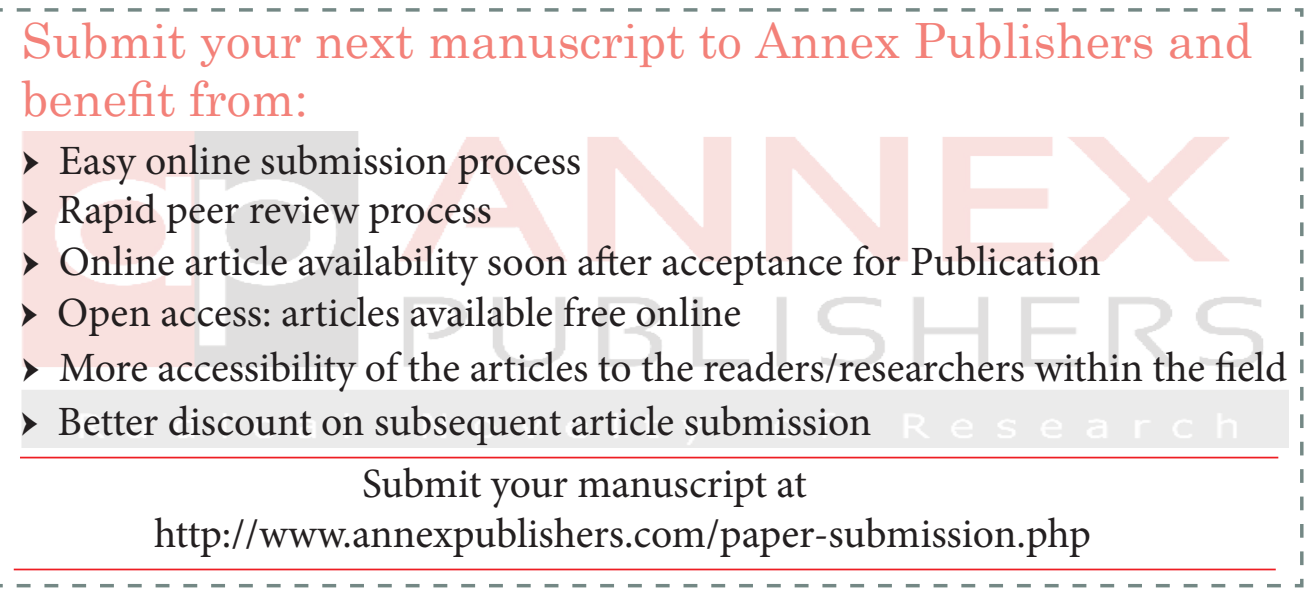

